\title{
Multiparametric MRI - local staging of prostate cancer and beyond
}

\author{
Iztok Caglic ${ }^{1,2}$, Viljem Kovac ${ }^{2,3}$, Tristan Barrett ${ }^{4,5}$ \\ ${ }^{1}$ Department of Radiology, Norfolk and Norwich University Hospital, Norwich, UK \\ ${ }^{2}$ Faculty of Medicine, University of Ljubljana, Slovenia \\ ${ }^{3}$ Institute of Oncology Ljubljana, Ljubljana, Slovenia \\ ${ }^{4}$ Department of Radiology, Addenbrooke's Hospital and University of Cambridge, Cambridge, UK \\ ${ }^{5}$ CamPARI Clinic, Addenbrooke's Hospital and University of Cambridge, Cambridge, UK
}

Radiol Oncol 2019; 53(2): 159-170.

Received 29 March 2019

Accepted 15 April 2019

Correspondence to: Assoc. Prof. Viljem Kovač, M.D., Ph.D., Institute of Oncology Ljubljana, Zaloška 2, 1000 Ljubljana, Slovenia. Phone: +386 15879 117; Fax: 38615879 400. E-mail: vkovac@onko-i.si

Disclosure: No potential conflicts of interest were disclosed.

Background. Accurate local staging is critical for treatment planning and prognosis in patients with prostate cancer (PCa). The primary aim is to differentiate between organ-confined and locally advanced disease with the latter carrying a worse clinical prognosis. Multiparametric MRI (mpMRI) is the imaging modality of choice for the local staging of PCa and has an incremental value in assessing pelvic nodal disease and bone involvement. It has shown superior performance compared to traditional staging based on clinical nomograms, and provides additional information on the site and extent of disease. MRI has a high specificity for diagnosing extracapsular extension (ECE), seminal vesicle invasion (SVI) and lymph node (LN) metastases, however, sensitivity remains poor. As a result, extended pelvic LN dissection remains the gold standard for assessing pelvic nodal involvement, and there has been recent progress in developing advanced imaging techniques for more distal staging.

Conclusions. T2W-weighted imaging is the cornerstone for local staging of PCa. Imaging at 3T and incorporating both diffusion weighted and dynamic contrast enhanced imaging can further increase accuracy. "Next generation" imaging including whole body MRI and PET-MRI imaging using prostate specific membrane antigen ( ${ }^{68} \mathrm{Ga}-\mathrm{PSMA}$ ), has shown promising for assessment of LN and bone involvement as compared to the traditional work-up using bone scintigraphy and body CT.

Key words: multiparametric MRl; prostate cancer; staging

\section{Introduction}

Accurate staging of prostate cancer is essential to inform prognosis and to stratify patients for appropriate management. MRI affords excellent soft tissue differentiation making it the most accurate modality for preoperative local T-staging of prostate cancer. ${ }^{1}$ According to European Association of Urology (EAU) guidelines, local staging investigations are only indicated for intermediate and highrisk patient groups. ${ }^{1}$ The high accuracy of multiparametric MRI (mpMRI) for detection of index lesions can aid T-staging, and can also identify tumours that may be missed by systematic biopsies, enabling early re-biopsy and accurate risk stratification. ${ }^{2}$

For the purposes of prognosis and management the primary aim is to differentiate organconfined disease from locally advanced disease. Extracapsular disease and seminal vesicle invasion carry a worse prognosis due to a greater risk of positive surgical margins leading to biochemical recurrence $^{3,4}$ and an increased risk of lymph node (LN) metastases, respectively. ${ }^{5}$ Nodal disease on its own is associated with a higher risk of progression to metastatic disease and thus a higher rate of cancer specific mortality. ${ }^{6-8}$ 
Traditionally, staging of prostate cancer has been performed using nomograms such as Partin tables which are based on digital rectal examination (DRE), prostate-specific antigen (PSA) levels, Gleason score and percentage core involvement as a surrogate of lesion volume. ${ }^{9-11}$ This approach often underestimates the true stage of the disease and has been shown to be inferior to $\mathrm{MRI}^{12}$, with the combination of MRI findings and nomograms showing significant added value for predicting adverse pathology in prostate cancer. ${ }^{13}$ In addition to improving accuracy, MRI also provides information on the site and extent of disease, which helps surgical planning, informing decision making on taking wider surgical margins to decrease the rate of positive margins, or performing nerve-sparing surgery to decrease morbidity. In case of a gross extracapsular extension or seminal vesicle invasion on MRI, external beam radiotherapy is a recom-

TABLE 1. Summary of TNM guidelines for the staging of prostate cancer

\begin{tabular}{|c|c|}
\hline Category & Definition \\
\hline \multicolumn{2}{|l|}{ Tumour } \\
\hline Tx & $\begin{array}{l}\text { Primary tumour cannot be assessed (e.g. CT study, severe } \\
\text { artefacts on MRI) }\end{array}$ \\
\hline Tla-Tlb & Tumour incidental histologic finding \\
\hline Tlc & Tumour identified by needle biopsy but not visible by imaging \\
\hline $\mathrm{T} 2$ & Organ confined disease \\
\hline T2a & The tumour involves up to one half of 1 side of the prostate \\
\hline $\mathrm{T} 2 \mathrm{~b}$ & The tumour involves more than one half of 1 side of the prostate \\
\hline $\mathrm{T} 2 \mathrm{C}$ & The tumour involves both sides of the prostate \\
\hline T3 & Extraprostatic extension \\
\hline T3a & $\begin{array}{l}\text { Extraprostatic extension (unilateral or bilateral) or microscopic } \\
\text { invasion of the bladder neck }\end{array}$ \\
\hline T3b & Tumour invades seminal vesicle(s) \\
\hline T4 & $\begin{array}{l}\text { Tumour invades adjacent structures other than seminal vesicles, } \\
\text { such as external sphincter, rectum, bladder, levator muscles, } \\
\text { and/or pelvic wall }\end{array}$ \\
\hline \multicolumn{2}{|l|}{ Node } \\
\hline Nx & Regional lymph nodes were not assessed \\
\hline NO & No positive regional lymph nodes \\
\hline N1 & Metastases in regional lymph node(s) \\
\hline \multicolumn{2}{|l|}{ Metastasis } \\
\hline$M x$ & M staging not assessed (e.g. MRI with pelvic only coverage) \\
\hline MO & No distant metastasis \\
\hline M1 & Distant metastasis \\
\hline Mla & Nonregional lymph node(s) \\
\hline Mlb & Bones \\
\hline Mlc & Other site(s) with or without bone disease \\
\hline
\end{tabular}

mended approach over brachytherapy or surgery, to avoid under dosing or positive surgical margins, respectively. ${ }^{14}$ As MR imaging currently does not offer sufficient diagnostic performance, extended pelvic lymph node dissection (ePLND) remains the gold standard for N-staging. ${ }^{1}$ However, ePLND has its own disadvantages including higher morbidity, with worse intraoperative and perioperative outcomes, and may result in under-sampling, thus its direct therapeutic effect is equivocal. ${ }^{15}$

This review aims to summarize the role of MRI in staging prostate cancer and focuses mainly on exploring the current evidence and providing a practical approach to assessment of extracapsular extension, seminal vesicle invasion and nodal disease.

\section{Staging of prostate cancer}

The most widely used system for staging of prostate cancer is the tumour, nodes, and metastases (TNM) staging system developed by the American Joint Committee on Cancer (AJCC). The current version of the TNM staging of prostate cancer (8th edition) was implemented in January 2018 introducing grade groups and simplifying organ-confined disease to pathological stage pT2 and omitting pT2a-pT2c, however, this sub-classification is retained for clinical staging (Table 1$).{ }^{16}$ In addition, Cancer-group staging of prostate cancer (stage IIV) is determined by TNM, PSA levels at diagnosis, and histologic Grade Groups. ${ }^{17}$

Locally confined disease (T1-T2) is further divided into stage $\mathrm{T} 1 \mathrm{a}$ and $\mathrm{T} 1 \mathrm{~b}$ tumours which are not apparent clinically and are found incidentally, typically at transurethral resection. From the radiological standpoint, the more relevant categories are stage T1c and T2 $(\mathrm{a}-\mathrm{c})$ as histologically they both represent a biopsy proven carcinoma albeit with an important difference: T1c cancer is by definition not visualised at MRI. This is relevant to active surveillance studies (AS) cohorts, with the term "non-visible lesion" (T1c) being introduced, based on the predictive nature of this feature, with a significantly increased progression free survival for non-visible lesions when compared with the MRIvisible lesions (T2). ${ }^{18}$

Locally advanced prostate cancer carries a worse prognosis that organ-confined disease. T3a disease describes extraprostatic extension, T3b seminal vesicle invasion, and $\mathrm{T} 4$ disease direct invasion of adjacent organs/structures (Table 1). In clinical practice those undergoing prostate mpMRI will 
have at least one sequence where the field of view covers the pelvis to the level of aortic bifurcation ${ }^{19}$ in order to evaluate the common iliac and bifurcation nodes (M1a) and from which partial M staging of the bony pelvis (M1b) can be performed.

\section{MR imaging}

\section{MRI scanners}

The Prostate Imaging-Reporting and Data System (PI-RADS) guidelines ${ }^{19}$ are aimed at standardizing MRI acquisition and interpretation and recommend MRI to be performed at 3T in order to increase signal-to-noise ratio (SNR) and spatial resolution, and decrease acquisition times. ${ }^{20,21}$ If acquisition protocols are optimized and contemporary technology is employed, then $1.5 \mathrm{~T}$ scanners are also able to provide sufficient diagnostic performance. 1.5T scanning may also be preferential when a patient has an implant non-compatible at higher field strengths, or with bilateral hip replacements in order to minimise artefact. ${ }^{22,23}$ The routine use of an endorectal coil (ERC) is no longer recommended..$^{19} 3 \mathrm{~T}$ scanners or contemporary $1.5 \mathrm{~T}$ scanners can provide sufficient imaging quality and although ERC increases SNR bring disadvantages, including deformation of the gland contour, near field coil flare, increased cost and time of examination as well as higher patient discomfort. ${ }^{19,21}$

\section{MRI protocol}

Standard prostate MRI protocols should incorporate anatomical T1W and T2W imaging in combination with the two functional sequences of diffusion weighted imaging (DWI) and dynamic contrast enhanced imaging (DCE). ${ }^{24} \mathrm{~A}$ set of minimal technical parameters for each of these sequences is outlined in Table $2^{25}$, although institutions are encouraged to optimize imaging protocols based on their own equipment, capacity and expertise. It is mandatory for axial T2W, DWI and DCE to be acquired in the same location, angle, slice thickness and gap to allow for synchronous scrolling through the images and direct evaluation of suspicious findings between the sequences. Axial T1WI is essential to assess post-biopsy haemorrhage, and is typically employed as the sequence to cover the pelvis to the aortic bifurcation to enable bone and nodal assessment.

$\mathrm{T} 2 \mathrm{~W}$ imaging is the key sequence for local T-staging of the prostate. The high in-plane spatial resolution allows for accurate evaluation of extra-
TABLE 2. PI-RADS $v 2.1$ recommended MR imaging protocols

\begin{tabular}{|c|c|}
\hline Imaging sequence & Technical parameters \\
\hline \multirow[t]{4}{*}{ T2 imaging } & $\begin{array}{l}\text { Axial plane and a minimum of one additional } \\
\text { orthogonal plane (either sagittal or coronal) } \\
\text { Straight axial plane to the patient or to the long axis of } \\
\text { the prostate }\end{array}$ \\
\hline & $\begin{array}{l}\text { FOV: } 12-20 \mathrm{~cm} \text { to image the entire prostate gland and } \\
\text { seminal vesicles }\end{array}$ \\
\hline & Section thickens/gap: $3 \mathrm{~mm} / 0 \mathrm{~mm}$ \\
\hline & $\begin{array}{l}\text { In-plane resolution: } \leq 0.7 \mathrm{~mm} \text { (phase) } x \leq 0.4 \mathrm{~mm} \\
\text { (frequency) }\end{array}$ \\
\hline \multirow[t]{8}{*}{ DW imaging } & Axial plane (same locations as for T2WI) \\
\hline & $\begin{array}{l}\text { Free-breathing spin echo EPI sequence combined with } \\
\text { spectral fat saturation is recommended }\end{array}$ \\
\hline & Section thickness/gap: $3 \mathrm{~mm} / 0 \mathrm{~mm}$ \\
\hline & TE: $\leq 90 \mathrm{~ms} ; \mathrm{TR}:>3000 \mathrm{~ms}$ \\
\hline & FOV: $16-22 \mathrm{~cm}$ \\
\hline & In plane dimension: $\leq 2.5 \mathrm{~mm}$ phase and frequency \\
\hline & $\begin{array}{l}\text { ADC map calculation: low b-value should be set at } 0- \\
100 \mathrm{~s} / \mathrm{mm} 2 \text {, high b-value should be }<1000 \mathrm{~s} / \mathrm{mm} 2\end{array}$ \\
\hline & $\begin{array}{l}\text { "High b-value": b-value of } \geq 1400 \mathrm{sec} / \mathrm{mm} 2 \text {; it can be } \\
\text { acquired by scanning or calculated }\end{array}$ \\
\hline \multirow[t]{9}{*}{ DCE } & Axial plane (same locations as for T2WI) \\
\hline & Fat suppression and/or subtraction is recommended \\
\hline & 2D or 3D T1 GRE sequence (preferred) \\
\hline & Section thickness/gap: $3 \mathrm{~mm} / 0 \mathrm{~mm}$ \\
\hline & Injection rate: $2-3 \mathrm{ml} / \mathrm{s}$ \\
\hline & TR/TE: $<100 \mathrm{~ms} /<5 \mathrm{~ms}$ \\
\hline & In-plane dimension: $\leq 2 \mathrm{~mm} \mathrm{X} \leq 2 \mathrm{~mm}$ \\
\hline & Temporal resolution: $\leq 15 \mathrm{~s}$ \\
\hline & Total observation: $>2$ min \\
\hline
\end{tabular}

$2 D=$ two-dimensional; $3 D=$ three-dimensional $; A D C=$ apparent diffusion coefficient; $E P I=$ echo planar imaging; $\mathrm{DW}=$ diffusion weighted; $\mathrm{FOV}=$ field of view; $\mathrm{GRE}=$ gradient echo $\mathrm{T} 2 \mathrm{~W}=\mathrm{T} 2$ weighted; TE = echo time; TR = repetition time

capsular extension, neurovascular bundle assessment and seminal vesicle invasion. Fast-spin-echo (FSE) or turbo-spin-echo (TSE) imaging should be obtained in the axial plane and in least one additional orthogonal plane (sagittal or coronal) with the highest quality possible and thin slices at $3 \mathrm{~mm}$ with no gap. ${ }^{25}$ 3D T2 weighted imaging with isotropic voxels and slice thickness at $1 \mathrm{~mm}$ may be obtained, with evidence suggesting utility for assessment of extraprostatic extension ${ }^{26}$ and for nodal and bone staging when combined with DWI of the entire pelvis (b-values $\left.0-1000 \mathrm{~s} / \mathrm{mm}^{2}\right){ }^{27}$

\section{Limitations}

Motion artefact. Bowel peristalsis is a well-known cause of motion artefact in abdominal imaging, 


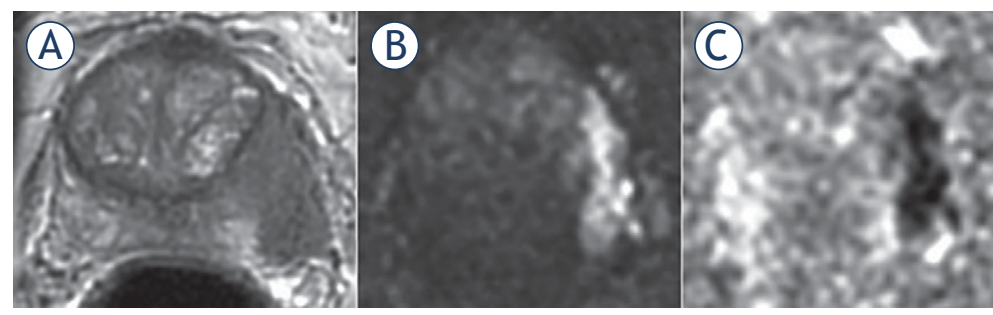

FIGURE 1. 65-yr-old man with PSA $19.5 \mathrm{ng} / \mathrm{ml}$. Invasion of the periprostatic fat and neurovascular bundle (NVB) infiltration at the left midgland consistent with T3a disease. Biopsy showed Gleason score (GS) $4+4=8$. Radical retropubic prostatectomy (RRP) confirmed GS $4+4=8$ and showed established T3a disease with a clear surgical margin (at least $1 \mathrm{~mm}$ ).

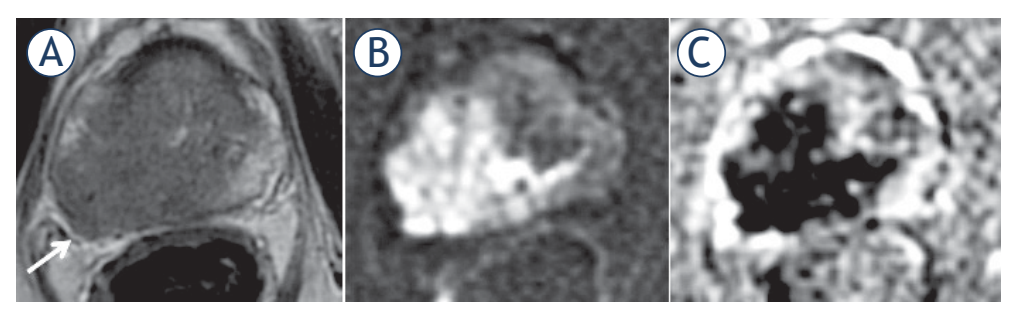

FIGURE 2. 77-yr-old man with PSA $38.2 \mathrm{ng} / \mathrm{ml}$. (A) T2 weighted (T2W) imaging; (B) diffusion weighted (DW) imaging: (C) apparent diffusion coefficient (ADC) map. T3a at the right mid gland with bulging and asymmetrical thickening of the right neurovascular bundle (arrow). Gleason score (GS) = 9 with extracapsular extension and clear surgical margins was confirmed at radical prostatectomy.

but the relatively low position of the prostate, remote from small bowel combined with limited evidence prior to PIRADS version 2 means that anti-spasmodic agents are not recommended in current guidelines. However, recent studies have shown use of anti-peristaltic agents significantly improves image quality of $\mathrm{T} 2 \mathrm{~W}$ imaging ${ }^{28,}{ }^{29}$ with better depiction of anatomical details (e.g. prostatic capsule and neurovascular bundles) as well as reducing non-diagnostic MRI to $<1 \%{ }^{29}$ Routine use of antiperistaltic agents (recommended dose $20 \mathrm{mg}$ HBB i.v. or $1 \mathrm{mg}$ glucagon) prior to prostate $\mathrm{mp}$ -

TABLE 3. PI-RADS $\vee 2$ criteria for predicting extraprostatic extension

Capsular abutment
Capsular irregularity, spiculation or retraction
Neurovascular bundle asymmetry or thickening
Obliteration of the rectoprostatic angle
Tumour-capsular contact $>10 \mathrm{~mm}$
Bulge or loss of capsule
Measurable extracapsular disease

MRI may therefore be beneficial for optimisation of $\mathrm{T} 2 \mathrm{~W}$ image quality, a key sequence of mpMRI for local staging. ${ }^{30}$ The risk of side-effects with these agents is low and these are usually minor and selflimiting. ${ }^{31}$

\section{T-staging}

\section{T3a disease}

Extension of the tumour into the periprostatic fat is defined as T3a disease, termed extracapsular extension (ECE). Of note, however, in a strict sense the prostate lacks a true capsule as an anatomic structure that encloses the gland but has rather an outer fibromuscular layer which is inseparable component of the prostatic stroma. ${ }^{32,33} \mathrm{~T} 3$ a disease also incorporates invasion into the neurovascular bundle, internal sphincter and bladder neck. ${ }^{1}$ Histopathologically, extracapsular extension (ECE) is sub-classified into focal and established with the latter carrying a worse prognosis. ${ }^{34}$ However, there is currently no clear consensus on the exact definitions of these, which can vary from a few glands beyond the capsule to cancer extending up to 0.5 $\mathrm{mm}$ radially from the capsule. ${ }^{1}$ In addition, focal ECE cannot be detected by MRI due to inherent resolution limits. ${ }^{35}$

Extracapsular extension has traditionally been evaluated by clinical criteria and nomograms such as Partin tables, which are based on PSA, DRE and Gleason score at biopsy. ${ }^{36}$ However, nomograms represent a patient level risk score alone, have been shown to be inferior to MRI ${ }^{11,37}$, and unlike MRI offer no information on location and extent of ECE. A meta-analysis by de Rooij et al. in 2016 showed MRI to have a high specificity of $91 \%$ but only moderate sensitivity at $57 \%$ in diagnosing ECE. Of note, this analysis included studies with both uni- and multiparametric protocols at both $1.5 \mathrm{~T}$ and $3 \mathrm{~T}$, and subanalysis of $3 \mathrm{~T}$ studies improved overall performance with specificity $86 \%$ and sensitivity $68 \% .38$ The main reasons for improvement being higher spatial resolution at 3T and improved lesion identification with a multiparametric approach, allowing interrogation of the capsule and neurovascular bundle in the adjacent region (Figure 2). ${ }^{39}$

Several approaches have been proposed and explored in order to increase diagnostic accuracy for the evaluation of ECE. Prostate imaging-reporting and data system (PI-RADS) guidelines recommends various morphologic criteria (Table 3$).{ }^{19}$ These have been evaluated and demonstrated sensitivity and specificity between $60 \%-81 \%$ and 
$75 \%-78 \%$, respectively, and showed moderate inter-reader agreement $(\mathrm{K}=0.45)$ for the prediction of T3a disease. ${ }^{40,41}$

In addition, the length of tumour contact with the capsule at MRI (Figure 3) has also been shown to be a strong predictor of $\mathrm{ECE}^{35,42,43}$ with good to excellent inter-reader agreement ${ }^{26,35}$ (Figure 3). ${ }^{26,35}$ However, a reliable threshold is yet to be established, with reported rates varying from $6-20 \mathrm{~mm}$, the PI-RADS v2 guidelines recommend an arbitrary threshold of $10 \mathrm{~mm}^{19}$, which pre-dates many of these studies. The reason for variability is likely multifactorial with different methodology employed and variations in scanner strength, vendor and protocols. For instance, Rosenkrantz et al. measured the length in a linear rather than curvilinear fashion which likely explains their lower reported threshold of $6 \mathrm{~mm} \cdot{ }^{35} \mathrm{In}$ addition, a more recent study suggests that thresholds differ between low(Grade Group 1-2) and high-grade (Grade Group $3-5)$ cancers, with the former having a positive predictive values (PPV) of $90.4 \%$ for ECE at $12.5 \mathrm{~mm}$ and the optimal cut-off for the latter being $5 \mathrm{~mm} .{ }^{26}$ This finding was further confirmed by Matsuoka et al. who reported significantly increased upstaging in low- versus high-grade cancers when the same threshold $(10 \mathrm{~mm})$ was applied. ${ }^{44}$ Given that lower apparent diffusion coefficient (ADC) values in prostate cancer correlate with higher Gleason score $^{45,46}$, this could potentially be exploited as an adjunct for more accurate diagnosis of T3a disease prior to biopsy results. To date however, there have been mixed results when applying ADC values for stage assessment, which may relate to difficulties in applying uniform quantitative values..$^{39,47-50}$

Another potential approach to improve sensitivity is utilisation of an isovolumetric 3D T2 imaging sequence to acquire thinner slices with less partial voluming and reformatting of isotropic images in multiple planes. Studies using 3D-T2 sequence have reported encouraging results with sensitivity and specificity ranging from $58.3 \%-84 \%$ and $73.1 \%-89 \%$, respectively. ${ }^{44,51-53}$ In addition, Caglic et al. proposed a new criterion of 3D Contact which significantly improved detection of ECE (sensitivity, specificity: $73.7 \%$ and $87.8 \%$ ) when compared to the length of capsular contact measured on conventional T2 imaging in axial plane (sensitivity, specificity: $59.6 \%, 87.8 \%) .{ }^{26}$ This approach exploited the reduced partial voluming due to thinner slices (Figure 4) and reconstruction of images in multiple planes in order to measure the more representative a truer length of capsular contact. Although not supported by work of Jäderling et

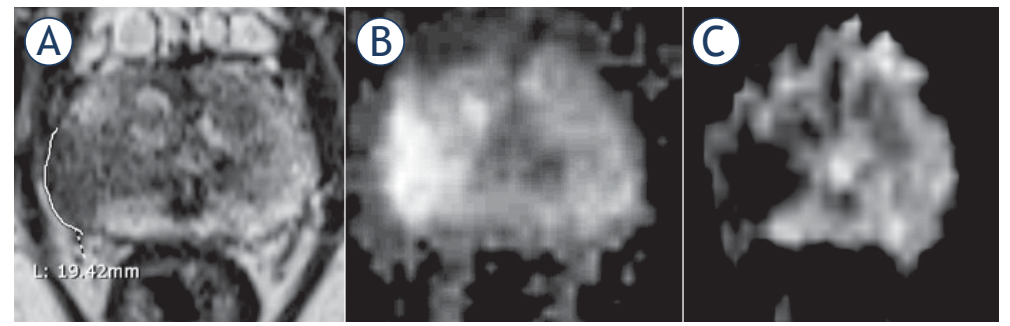

FIGURE 3. 74-yr-old man with PSA $35.2 \mathrm{ng} / \mathrm{ml}$. (A) T2 weighted (T2W) imaging, (B) diffusion weighted (DW) imaging, (C) apparent diffusion coefficient (ADC) map. T3a at the right mid gland as suggested by a broad capsular contact at $19.4 \mathrm{~mm}$. Biopsy showed Gleason score (GS) $4+4=8$ disease. Patient underwent radiotherapy.
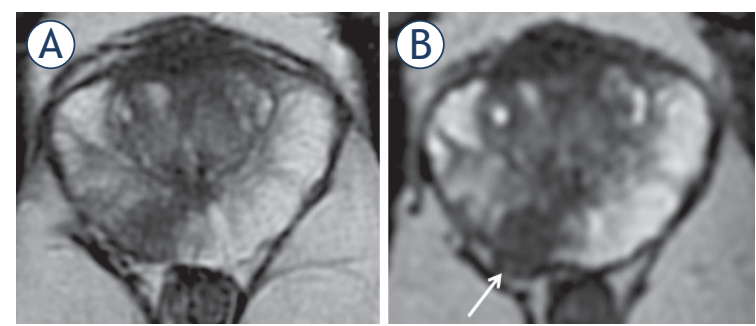

FIGURE 4. 57-yr-old man with PSA $26 \mathrm{ng} / \mathrm{ml}$. (A) Axial T2 weighted imaging (T2WI) shows mid gland right peripheral zones (PZ) lesion (arrow) with capsular contact but no tumour extension beyond it. (B) axial thin-sliced cube reformat suggests capsular breach and right neurovascular bundle involvement (arrow). Prostatectomy showed tumour in the right mid gland, Gleason score $4+5=9$, with established extracapsular extension (ECE) (pT3a).

al. using 3D-T2 reconstructions, it should be noted that their analysis was based on morphological criteria and not on quantifying capsular contact. ${ }^{54}$

Although diagnostic accuracy for early ECE is improving, sensitivity remains relatively poor, and it should be noted that these results come from experienced centres, utilizing modern equipment and optimised protocols. As a result, equivocal MRI findings should not change the planned treatment course, but rather ensure discussion between radiologists and urologists at multidisciplinary meetings on a case-by-case basis. Practical advice would be to flag indeterminate features of ECE, to allow wider surgical margins to be taken in the corresponding region..$^{10}$ Furthermore, reporting the exact location of T3a disease is important, as clear margins are harder to obtain at the apex whereas tumours remote from the neurovascular bundle (NVB) such as in the anterior location will allow nerve sparing surgery and reduce resultant morbidity from urinary incontinence or erectile dysfunction. 


\section{T3b disease}

T3b disease is defined as involvement of one or both seminal vesicles (SV) by prostate cancer, with the prevalence of SV invasion in surgical series being reported at $4-23 \% .{ }^{55,56}$ Patients with T3b disease carry an increased risk of lymph node involvement, local recurrence and distant metastases ${ }^{57}$, making preoperative identification of SV involvement an important factor for prognosis and treatment planning. Patients with T3b disease are typically not offered radical prostatectomy or brachytherapy unless as part of a multimodal approach, and are usually offered external beam radiotherapy (EBRT) and androgen deprivation therapy (ADT).

MRI has been shown to outperform clinical risk assessment tools such as Kattan nomogram and Partin tables in predicting SV involvement ${ }^{13,58}$, with meta-analyses showing moderate sensitivity of $73 \%$ and specificity of $95 \%$ for multiparametric MRI studies at 3T. ${ }^{38}$ Recent work by Grivas et al. including 527 patients at 3T mpMRI achieved similar results with sensitivity, specificity, PPV and negative predictive values (NPV) at $75.9 \%, 94.7 \%, 62 \%$ and $97 \%$, respectively. ${ }^{59}$
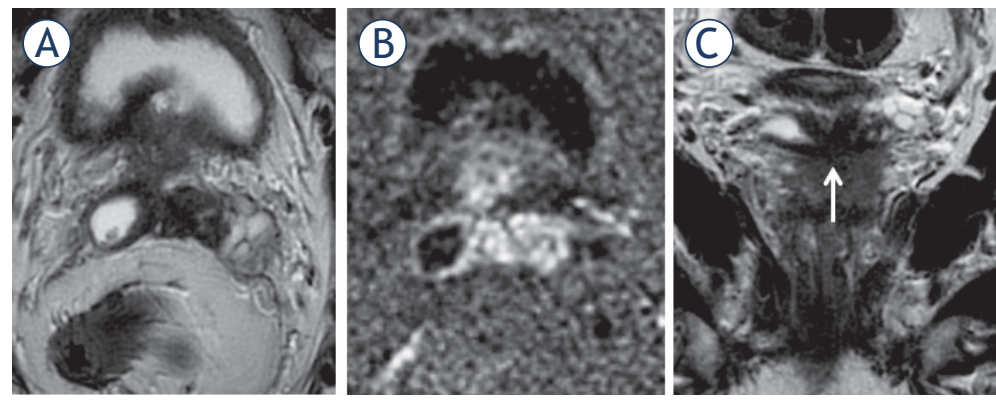

FIGURE 5. 65-yr-old-man with PSA $=15.3 \mathrm{ng} / \mathrm{ml}$ and Gleason score $4+4=8$ at biopsy. Axial (A) and coronal (C) T2 weighted imaging (T2WI) and diffusion weighted imaging (DWI) (B). T3b involving both seminal vesicles via ejaculatory ducts, shown in the coronal plane (arrow).
Histopathologically, SV invasion is defined as prostate cancer penetrating the SV muscular wall, with tumour involving the extraprostatic portion of the vesicles rather than the intraprostatic ejaculatory ducts. ${ }^{57}$ Three routes of invasion have been described, Type I: direct spread via the ejaculatory duct complex (Figure 5), Type II: extracapsular spread of disease with invasion via the outer seminal vesicle wall and type III: metastatic involvement from a remote intraprostatic lesion (Figure 6). The first two types individually or in combination account for more than $95 \%$ of cases, with type III spread being extremely rare. ${ }^{60,61}$

Seminal vesicles are best evaluated on T2W imaging in combination with functional imaging. Coronal or sagittal reformats are especially useful in demonstrating the type of spread. In Type I invasion, invasion via the erectile dysfunction (ED) causes SV expansion with a low signal intraluminal mass and may cause diffuse or focal wall thickening. In Type II involvement, there is obliteration of the angle between the base of the prostate and SV. ${ }^{60}$ In addition, in 2009 Jung et al. proposed a novel six-tier classification system for SV invasion based on morphological appearance of SV on $\mathrm{T} 2 \mathrm{~W}$ imaging (Class $0=$ normal SV appearance, Class $5=$ apparent mass lesion with destructive architecture) showing sensitivity and specificity of 71.4 and $96.6 \%$, respectively. ${ }^{62}$ More recent work incorporating functional sequences has further increased accuracy, with DWI proving to be of more incremental value than DCE. ${ }^{56,63}$

There are known pitfalls to be aware of when assessing for SV involvement, such as diffuse wall thickening due to SV atrophy or asymmetry. In addition, there can be large variation with a mean right - left asymmetry of $20 \%$ and surgical series suggesting SV length between sides can vary up to 9 -fold. ${ }^{64}$ Post-biopsy haemorrhage can mimic the low T2 signal of prostate cancer and review
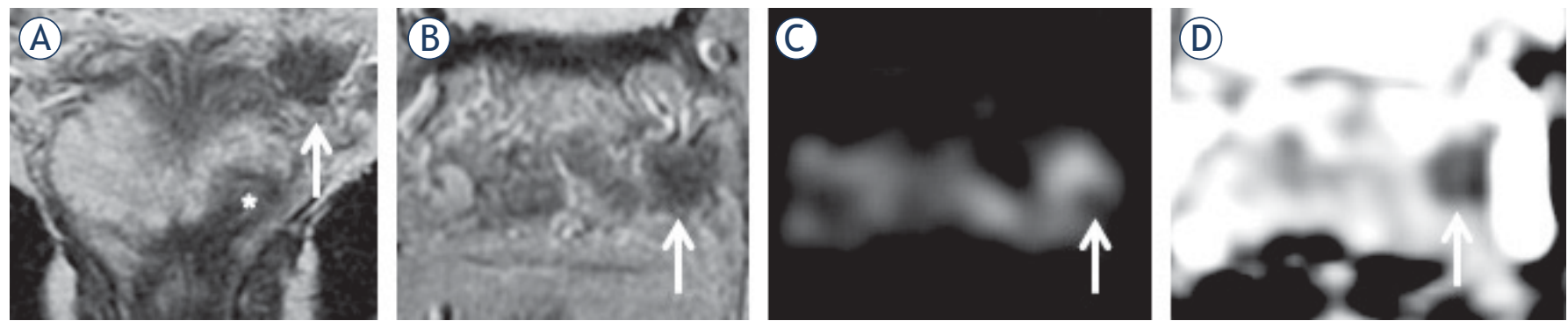

FIGURE 6. 67-yr-old man with raising PSA $=12.7 \mathrm{ng} / \mathrm{ml}$. (A) (coronal) and (B) (axial) T2 weighted (T2W) imaging shows index lesion in the left apex (*) and a low signal focus in the left seminal vesicle (arrow) with corresponding restricted diffusion on diffusion weighted imaging (DWI) ((C); arrow) and apparent diffusion coefficient (ADC) map ((D); arrow). 

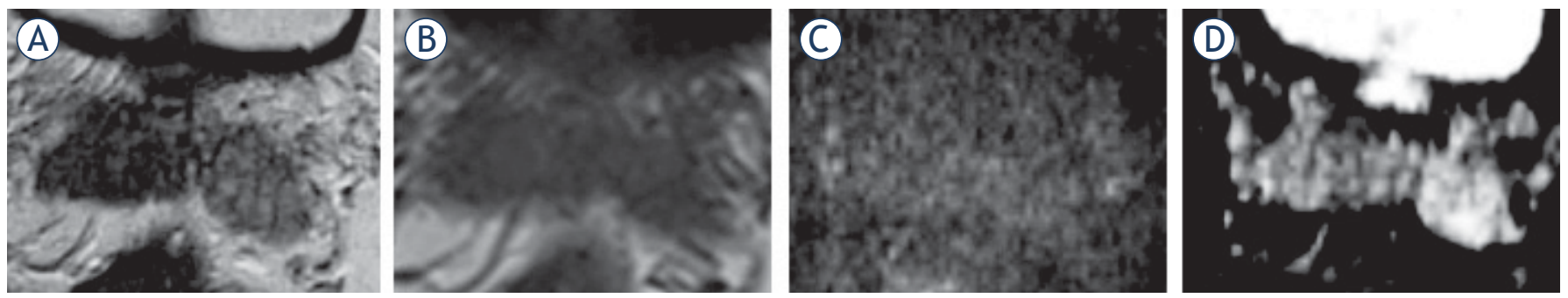

FIGURE 7. 55-yr-old-man with PSA $32 \mathrm{ng} / \mathrm{ml}$. (A) T2 weighted (T2W) imaging, (B) T1 weighted (TIW) imaging, (C) diffusion weighted (DW) imaging, (D) apparent diffusion coefficient (ADC) map. Low T2 signal in the right seminal vesicle is mimicking prostate cancer (D), however, there is no restricted diffusion. Biopsy of the right seminal vesicle showed amyloidosis which was confirmed at radical prostatectomy. Index tumour with Gleason score $4+$ $3=7$ was in the left peripheral zone.

of T1W imaging for high signal within the SV is therefore essential. Another important mimic of prostate cancer is amyloidosis which exhibits low T2 signal but does not show diffusion restriction (Figure 7). In cases with equivocal SV findings on prebiopsy MRI, patients can undergo subsequent targeted biopsy in order to correctly stage the disease (Figure 7) ${ }^{65}$

Although PI-RADS v2 does not recommend abstinence from ejaculation prior to prostate $\mathrm{mpM}$ $\mathrm{RI}^{19}$, some centres require patients to refrain from ejaculation prior to imaging in order to achieve maximal distension. Recent studies suggest 72 hours abstinence as the recommended interval to achieve maximal seminal vesicle distension. ${ }^{66-69}$ This may be beneficial in evaluation of seminal vesicle invasion but further prospective studies including patients with prostate cancer are required to determine the effect on local staging accuracy.

\section{$\mathrm{N}$-Staging}

EAU guidelines recommend N-staging should be performed on prebiopsy MRI in patients from all risk groups. ${ }^{1}$ This is in line with PI-RADS v2 guidelines which recommend that the prostate MRI protocol, which is primarily aimed at evaluating gland-confined and locally advanced disease, should also incorporate an additional sequence for the purpose of pelvic nodal staging from the level of aortic bifurcation. ${ }^{24}$ PCa spreads primarily to four pelvic nodal stations, considered regional nodes: the obturator, internal and external iliac and presacral LNs. Involvement of any regional node is classified as N1 stage, whilst involvement of nonregional stations (paraaortic or paracaval LNs) represents M1a disease. ${ }^{17,70}$ Nodal mapping studies have shown that approximately $75 \%$ of pelvic nodal metastases are distributed between the ob- turator fossa, internal and external iliac chain and the remaining $25 \%$ between the presacral, common iliac or aortic bifurcation group..$^{71,72}$

MRI has traditionally relied on size and morphological criteria in LN assessment including an enlarged size $(>8 \mathrm{~mm})$, loss of fatty hilum, rounded shape, low T2W signal similar to primary tumour, or an irregular border. This is of limited diagnostic accuracy mainly due to low sensitivity, with a meta-analysis from 2008 incorporating anatomical imaging studies alone (T2W and $\mathrm{T} 1 \mathrm{~W}$ ) reporting a sensitivity of only $39 \%$ (specificity $82 \%$ ). ${ }^{73}$ Size criteria in isolation is unhelpful, with a recent study showing the majority $(68 \%)$ of metastatic nodes to have a short axis diameter $<5 \mathrm{~mm} \cdot{ }^{27}$ More recent studies have tried to establish whether an ADC threshold can be applied for discrimination of benign from malignant LNs.27,74-78 Although malignant LNs typically exhibit lower ADC values, there is significant overlap between normal and pathological LNs as well as large variation in the reported thresholds, limiting the value of quantitative ADC measures at an individual patient level..$^{79}$ Reasons for poor discrimination include micro metastasis being unlikely to lower the overall ADC value of a node, whilst some benign conditions (lipomatosis, sinus histiocytosis, and follicular hyperplasia) ${ }^{27}$ as well as inflammation (sarcoidosis and catch scratch disease) can also result in restricted diffusion within LNs. ${ }^{80,81}$ In addition, reproducibility of ADC measurements in small structures such as LNs may be insufficient and differences in acquisition protocols between centres further inhibits establishment of an absolute threshold. ${ }^{82-85}$ Consequently, some studies have focused on qualitative assessment of DWI using high $b$-value imaging in combination with anatomical T2W and reported improved performance with sensitivities of $55-73 \%$ and specificities of $86-90 \% .{ }^{27,86}$ Normal LNs have an inherent relatively long T2 relaxation time and will there- 
fore appear as high signal intensity structures on high b-value imaging (Figure 8), which is especially useful in depicting LNs as a "nodal map" when these do not meet size criteria. Detected nodes should then be carefully evaluated on T2W imaging in order to avoid false positive results due to structures which also appear bright on high bvalue DWI (bowel mucosa, vessels, nerves) ${ }^{87}$ and to assess morphological features of malignancy.

Current diagnostic performance of MRI in nodal staging is sub-optimal, thus ePLND remains the gold standard. Due to the limited sensitivity (high false negative rate) of MRI, negative findings should not deter surgeons from performing lymphadenectomy in patients with a high clinical risk for LN involvement. Conversely, the specificity of MRI is high (low false positive rate) and LNs considered to be suspicious at MRI warrant resection.

Further work and development of imaging techniques with a high diagnostic performance is needed in order to more efficiently and less invasively identify patients with metastatic LNs. Initial clinical trials with prostate specific membrane antigen $\left({ }^{68} \mathrm{Ga}-\mathrm{PSMA}\right)$ PET-MRI have shown promising results for detection of LN metastases ${ }^{88,89}$, resulting in change of treatment (either to systemic treat-
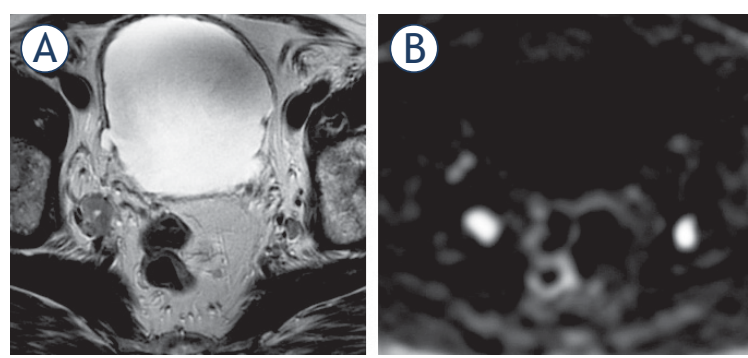

FIGURE 8. 77-yr-old man with PSA $=38.2 \mathrm{ng} / \mathrm{ml}$. Enlarged nodes bilaterally consistent with metastatic involvement on T2 weighted imaging (T2WI) (A), more conspicuous on diffusion weighted imaging (DWI) (B).
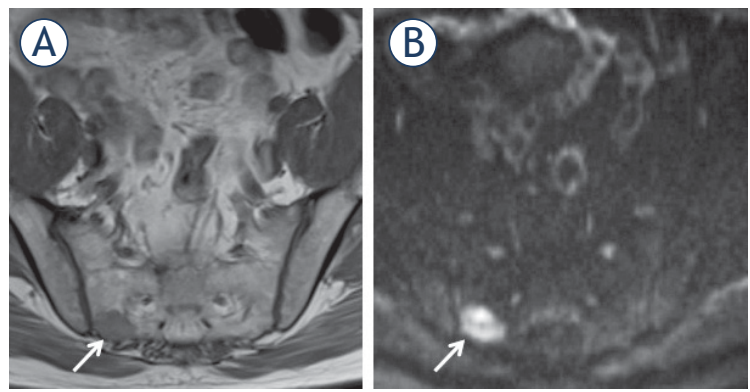

FIGURE 9. 61-yr-old man with PSA $=12.7 \mathrm{ng} / \mathrm{ml}$. Bone metastasis (arrow) in the right sacrum shown as low signal on $\mathrm{Tl}$ weighted (TIW) imaging (A); more conspicuous as high signal on diffusion weighted imaging (DWI) (B). ment or active surveillance) in approximately one third of patients. ${ }^{90} \mathrm{MR}$ lymphangiography (MRL) with ultra-small superparamagnetic iron oxide (USPIO) has also demonstrated encouraging results with studies reporting sensitivity of $65-100 \%$ and specificity of $93-100 \%$ on a per patient basis. ${ }^{91-93}$ However, USPIO is currently not licenced for general clinical use, with only the Netherlands producing it (commercially known as Combidex) and licensing it mainly for the research purposes in patients with PCa. ${ }^{94}$

\section{M-Staging}

EAU guidelines recommend staging for metastatic disease (M1a-M1c) in patients with unfavourable intermediate (International Society of Urological Pathology [ISUP] grade group 3) or high-risk (ISUP grade group 4-5) disease. ${ }^{1}$ Current guidelines recommend evaluation of non-regional LNs and visceral metastases (M1a and M1c disease, respectively) by CT abdomen/pelvis imaging, combined with bone scintigraphy (BS) for evaluation of bone metastases (M1b disease) (Figure 9). ${ }^{95}$

Several studies have shown MRI (either wholebody MRI or axial skeleton only MRI) to significantly outperform BS for assessment of M1b disease, with a thorough meta-analysis from 2014 reporting MRI sensitivity and specificity to be $97 \%$ and $95 \%$ compared to BS at $79 \%$ and $82 \%$, respectively. ${ }^{96} \mathrm{MRI}$ is not incorporated into current guidelines mainly due to its limited availability and lower cost effectiveness. ${ }^{96}$ However, over the last decade whole body MRI (WB-MRI) has been gradually gaining attention due to its ability to detect bone marrow infiltration by malignant cells before bone remodelling occurs and therefore becomes visible on BS. ${ }^{97}$ The METastasis Reporting and Data System for Prostate (MET-RADS-P) is aimed at practical guidance for acquisition, interpretation, and reporting of WB-MRI in advanced prostate cancer. ${ }^{98}$ The recommended protocol consists of a combination of anatomical and functional sequences (T1W, short tau inversion recovery [STIR] or fat suppressed T2W and DW imaging). Bone metastases appear as low signal on T1W imaging, bright on STIR or fat suppressed T2W and with restricted diffusion. Beside bone assessment, WB-MRI can also provide N-staging and assess for involvement of visceral organs. ${ }^{99,100}$ Whilst the diagnostic potential of WBMRI is promising, there are barriers to widespread adoption, including additional coils required, increased scanning time, the 
need for sub-specialised knowledge, and increased reporting time. A recent study from 2018 by Larbi et al. has shown a possible means of overcoming some of these disadvantages by demonstrating that the combination of either T1-DWI or T1-STIR is non-inferior to a full protocol (Figure 9), whilst at the same time showing good interobserver agreement. ${ }^{101}$

\section{Conclusions}

MpMRI is the recommended modality for the local staging of prostate cancer. It has shown superior performance compared to traditional staging based on clinical nomograms, and provides additional information on the site and extent of disease. T2Wweighted imaging remains the cornerstone for ECE and SV invasion assessment, however, improved accuracy can be achieved by scanning on 3T devices with the incorporation of diffusion weighted and dynamic contrast enhanced imaging. Whilst its role in nodal and bone staging outside academic centres is currently limited, there are emerging "next generation" imaging modalities including ${ }^{68}$ Ga-PSMA-PET/MRI and whole-body MRI offer potential to become the future standard of care for evaluation, having shown superior results for distal staging in comparison to the traditional workup with bone scintigraphy and body CT. Despite the advantages of mpMRI there remain limitations which should be known to radiologists and other members of the multidisciplinary team in order to jointly decide on the best treatment options for prostate cancer patients on an individual basis.

\section{Acknowledgements}

The authors acknowledge grant support from the Evelyn Trust UK and research support from Cancer Research UK, National Institute of Health Research Cambridge Biomedical Research Centre, Cancer Research UK and the Engineering and Physical Sciences Research Council Imaging Centre in Cambridge and Manchester and the Cambridge Experimental Cancer Medicine Centre.

\section{References}

1. Mottet N, van den Bergh RCN, Briers E, Bourke L, Cornford P, De Santis M, et al. EAU - ESTRO - ESUR - SIOG guidelines on prostate cancer 2018. In: European Association of Urology guidelines 2018 Edition. Arnhem, The Netherlands: European Association of Urology Guidelines Office; 2018.
2. Brizmohun Appayya M, Adshead J, Ahmed HU, Allen C, Bainbridge A, Barrett $T$, et al. National implementation of multi-parametric magnetic resonance imaging for prostate cancer detection - recommendations from a UK consensus meeting. BJU Int 2018; 122: 13-25. doi: 10.1111/bju.14361

3. Swanson GP, Riggs M, Hermans M. Pathologic findings at radical prostatectomy: risk factors for failure and death. Urol Oncol 2007; 25: 110-4. doi: 10.1016/j.urolonc.2006.06.003

4. Godoy G, Tareen BU, Lepor H. Site of positive surgical margins influences biochemical recurrence after radical prostatectomy. BJU Int 2009; 104: 1610-4. doi: 10.1111/j.1464-410X.2009.08688.x

5. Epstein JI, Partin AW, Potter SR, Walsh PC. Adenocarcinoma of the prostate invading the seminal vesicle: prognostic stratification based on pathologic parameters. Urology 2000; 56: 283-8. doi: 10.1016/S0090-4295(00)006403

6. Cagiannos I, Karakiewicz P, Eastham JA, Ohori M, Rabbani F, Gerigk C, et al. A preoperative nomogram identifying decreased risk of positive pelvic lymph nodes in patients with prostate cancer. J Urol 2003; 170: 1798-803. doi: 10.1097/01.ju.0000091805.98960.13

7. Gervasi LA, Mata J, Easley JD, Wilbanks JH, Seale-Hawkins C, Carlton CE, et al. Prognostic significance of lymph nodal metastases in prostate cancer. J Urol 1989; 142: (2 Part 1)332-6. doi: 10.1016/S0022-5347(17)38748-7

8. Eggener SE, Scardino PT, Walsh PC, Han M, Partin AW, Trock BJ, et al. Predicting 15-year prostate cancer specific mortality after radical prostatectomy. J Urol 2011; 185: 869-75. doi: 10.1016/j.juro.2010.10.057

9. Morlacco A, Sharma V, Viers BR, Rangel L, Carlson RE, Froemming AT, et al. The incremental role of magnetic resonance imaging for prostate cancer staging before radical prostatectomy. Eur Urol 2017; 71: 701-4. doi: 10.1016/j.eururo.2016.08.015

10. Ward JF, Slezak JM, Blute ML, Bergstralh EJ, Zincke H. Radical prostatectomy for clinically advanced (cT3) prostate cancer since the advent of prostatespecific antigen testing: 15-year outcome. BJU Int 2005; 95: 751-6. doi: 10.1111/j.1464-410X.2005.05394.x

11. Augustin H, Fritz GA, Ehammer T, Auprich M, Pummer K. Accuracy of 3-Tesla magnetic resonance imaging for the staging of prostate cancer in comparison to the partin tables. Acta Radiol 2009; 50: 562-9. doi: $10.1080 / 02841850902889846$

12. Gupta RT, Faridi KF, Singh AA, Passoni NM, Garcia-Reyes K, Madden JF, et al. Comparing 3-T multiparametric MRI and the Partin tables to predict organconfined prostate cancer after radical prostatectomy. Urol Oncol Semin Orig Investig 2014; 32: 1292-9. doi: 10.1016/j.urolonc.2014.04.017

13. Rayn KN, Bloom JB, Gold SA, Hale GR, Baiocco JA, Mehralivand S, et al. Added value of multiparametric magnetic resonance imaging to clinical nomograms for predicting adverse pathology in prostate cancer. J Urol 2018; 200: 1041-7. doi: 10.1016/j.juro.2018.05.094

14. Boehmer D, Maingon P, Poortmans P, Baron M-H, Miralbell R, Remouchamps $\mathrm{V}$, et al. Guidelines for primary radiotherapy of patients with prostate cancer. Radiother Oncol 2006; 79: 259-69. doi: 10.1016/j.radonc.2006.05.012

15. Mottet N, van der Poel HG, Rouvière O, Matveev VB, Schoots IG, Briers $E$, et al. The benefits and harms of different extents of lymph node dissection during radical prostatectomy for prostate cancer: a systematic review. Eur Urol 2017; 72: 84-109. doi: 10.1016/j.eururo.2016.12.003

16. Buyyounouski MK, Choyke PL, McKenney JK, Sartor O, Sandler HM, Amin $\mathrm{MB}$, et al. Prostate cancer - major changes in the American Joint Committee on Cancer eighth edition cancer staging manual. CA Cancer J Clin 2017; 67: 245-53. doi: 10.3322/caac. 21391

17. Amin MB, Edge SB, Greene FL, Byrd DR, Brookland RK, Washington MK, et al, editors. AJCC cancer staging manual. 8th edition. New York: Springer; 2017.

18. Giganti F, Moore CM, Punwani S, Allen C, Emberton M, Kirkham A. The natural history of prostate cancer on MRI: lessons from an active surveillance cohort. Prostate Cancer Prostatic Dis 2018; 21: 556-63. doi: 10.1038/ s41391-018-0058-5

19. Weinreb JC, Barentsz JO, Choyke PL, Cornud F, Haider MA, Macura KJ, et al. PI-RADS prostate imaging - reporting and data system: 2015, Version 2. Eur Urol 2016; 69: 16-40. doi: 10.1016/j.eururo.2015.08.052

20. Rouvière $\mathrm{O}$, Hartman RP, Lyonnet $\mathrm{D}$. Prostate $M R$ imaging at high-field strength: evolution or revolution? Eur Radiol 2006; 16: 276-84. doi: org/10.1007/s00330-005-2893-8 
21. Turkbey B, Merino MJ, Gallardo EC, Shah V, Aras O, Bernardo M, et al Comparison of endorectal coil and nonendorectal coil T2W and diffusionweighted MRI at 3 Tesla for localizing prostate cancer: correlation with whole-mount histopathology. J Magn Reson Imaging 2014; 39: 1443-8. doi: 10.1002/jmri.24317

22. Czarniecki M, Caglic I, Grist JT, Gill AB, Lorenc K, Slough RA, et al. Role of PROPELLER-DWI of the prostate in reducing distortion and artefact from total hip replacement metalwork. Eur J Radiol 2018; 102: 213-9. doi: org/10.1016/j.ejrad.2018.03.021

23. Gill $A B$, Czarniecki M, Gallagher FA, Barrett T. A method for mapping and quantifying whole organ diffusion-weighted image distortion in MR imaging of the prostate. Sci Rep 2017; 7: 12727. doi: 10.1038/s41598017-13097-6

24. Barrett T, Turkbey B, Choyke PL. PI-RADS version 2: what you need to know. Clin Radiol 2015; 70: 1165-76. doi: 10.1016/j.crad.2015.06.093

25. Turkbey B, Rosenkrantz AB, Haider MA, Padhani AR, Villeirs G, Macura KJ, et al. Prostate Imaging Reporting and Data System Version 2.1: 2019 update of Prostate Imaging Reporting and Data System Version 2. Eur Urol 2019; 0232: 1-12. doi: 10.1016/j.eururo.2019.02.033

26. Caglic I, Povalej Brzan P, Warren AY, Bratt O, Shah N, Barrett T. Defining the incremental value of 3D T2-weighted imaging in the assessment of prostate cancer extracapsular extension. Eur Radiol 2019. [Epub ahead of print]. doi: 10.1007/s00330-019-06070-6

27. Thoeny HC, Froehlich JM, Triantafyllou M, Huesler J, Bains $\sqcup$, Vermathen $P$, et al. Metastases in normal-sized pelvic lymph nodes: detection with diffusion-weighted MR imaging. Radiology 2014; 273: 125-35. doi: 10.1148/ radiol.14132921

28. Slough RA, Caglic I, Hansen NL, Patterson AJ, Barrett T. Effect of hyoscine butylbromide on prostate multiparametric MRI anatomical and functional image quality. Clin Radiol 2018; 73: 216.e9-14. doi: 10.1016/j. crad.2017.07.013

29. Ullrich T, Quentin M, Schmaltz AK, Arsov C, Rubbert C, Blondin D, et al. Hyoscine butylbromide significantly decreases motion artefacts and allows better delineation of anatomic structures in $\mathrm{mp}-\mathrm{MRI}$ of the prostate. Eur Radiol 2018; 28: 17-23. doi: org/10.1007/s00330-017-4940-7

30. Caglic I, Barrett T. Optimising prostate mpMRI: prepare for success. Clin Radiol 2019. [Epub ahead of print]. doi: 10.1016/j.crad.2018.12.003

31. Dyde R, Chapman AH, Gale R, Mackintosh A, Tolan DJM. Precautions to be taken by radiologists and radiographers when prescribing hyoscine- $\mathrm{N}$-butylbromide. Clin Radio/ 2008; 63: 739-43. doi: org/10.1016/j.crad.2008.02.008

32. Ayala AG, Ro JY, Babaian R, Troncoso P, Grignon DJ. The prostatic capsule. Am J Surg Pathol 1989; 13: 21-7. doi: 10.1097/00000478-19890100000003

33. McNeal JE. Normal histology of the prostate. Am J Surg Pathol 1988; 12 619-33. doi: 10.1097/00000478-198808000-00003

34. Ball MW, Partin AW, Epstein Jl. Extent of extraprostatic extension independently influences biochemical recurrence-free survival: evidence for further PT3 subclassification. Urology 2015; 85: 161-4. doi: org/10.1016/j. urology.2014.08.025

35. Rosenkrantz AB, Shanbhogue AK, Wang A, Kong MX, Babb JS, Taneja SS. Length of capsular contact for diagnosing extraprostatic extension on prostate MRI: assessment at an optimal threshold. J Magn Reson Imaging 2016; 43: 990-7. doi: 10.1002/jmri.25040

36. Eifler JB, Feng Z, Lin BM, Partin MT, Humphreys EB, Han M, et al. An updated prostate cancer staging nomogram (Partin tables) based on cases from 2006 to 2011. BJU Int 2013; 111: 22-9. doi: 10.1111/j.1464410X.2012.11324.X

37. Turkbey B, Mani H, Aras O, Ho J, Hoang A, Rastinehad AR, et al. Prostate cancer: can multiparametric MR imaging help identify patients who are candidates for active surveillance? Radiology 2013; 268: 144-52. doi: 10.1148/radiol.13121325

38. de Rooij M, Hamoen EHJ, Witjes JA, Barentsz JO, Rovers MM. Accuracy of magnetic resonance imaging for local staging of prostate cancer: a diagnostic meta-analysis. Eur Urol 2016; 70: 233-45. doi: 10.1016/j. eururo.2015.07.029
39. Lawrence EM, Gallagher FA, Barrett T, Warren AY, Priest AN, Goldman DA, et al. Preoperative 3-T diffusion-weighted MRI for the qualitative and quantitative assessment of extracapsular extension in patients with intermediate - or high-risk prostate cancer. AJR Am J Roentgenol 2014; 203: W280-6. doi: 10.2214/AJR.13.11754

40. Boesen L, Chabanova E, Løgager V, Balslev I, Mikines K, Thomsen HS. Prostate cancer staging with extracapsular extension risk scoring using multiparametric MRI: a correlation with histopathology. Eur Radiol 2015; 25: 1776-85. doi: 10.1007/s00330-014-3543-9

41. Schieda N, Quon JS, Lim C, El-Khodary M, Shabana W, Singh V, et al. Evaluation of the European Society of Urogenital Radiology (ESUR) PI-RADS scoring system for assessment of extra-prostatic extension in prostatic carcinoma. Eur J Radiol 2015; 84: 1843-8. doi: 10.1016/j.ejrad.2015.06.016

42. Baco E, Rud E, Vlatkovic L, Svindland A, Eggesb $\varnothing H B$, Hung AJ, et al. Predictive value of magnetic resonance imaging determined tumor contact length for extracapsular extension of prostate cancer. J Urol 2015; 193: 466 72. doi: 10.1016/j.juro.2014.08.084

43. Woo S, Kim SY, Cho JY, Kim SH. Length of capsular contact on prostate MRI as a predictor of extracapsular extension: which is the most optimal sequence? Acta Radiol 2017: 58: 489-97. doi: 10.1177/0284185116658684

44. Matsuoka Y, Ishioka J, Tanaka H, Kimura T, Yoshida S, Saito K, et al. Impact of the Prostate Imaging Reporting and Data System, Version 2, on MRI diagnosis for extracapsular extension of prostate cancer. AJR Am J Roentgenol 2017; 209: W76-84. doi: org/10.2214/AJR.16.17163

45. Hambrock T, Somford DM, Huisman HJ, van Oort IM, Witjes JA, Hulsbergenvan de Kaa CA, et al. Relationship between apparent diffusion coefficients at 3.0-T MR imaging and Gleason grade in peripheral zone prostate cancer. Radiology 2011; 259: 453-61. doi: 10.1148/radiol.11091409

46. Verma S, Rajesh A, Morales $H$, Lemen L, Bills $G$, Delworth $M$, et al. Assessment of aggressiveness of prostate cancer: correlation of apparent diffusion coefficient with histologic grade after radical prostatectomy. AJR Am J Roentgenol 2011; 196: 374-81. doi: 10.2214/AJR.10.4441

47. Kim CK, Park SY, Park JJ, Park BK. Diffusion-weighted MRI as a predictor of extracapsular extension in prostate cancer. AJR Am J Roentgenol 2014; 202: W270-6. doi: 10.2214/AJR.13.11333

48. Woo S, Cho JY, Kim SY, Kim SH. Extracapsular extension in prostate cancer: added value of diffusion-weighted MRI in patients with equivocal findings on T2-weighted imaging. AJR Am J Roentgenol 2015; 204: W168-75. doi: 10.2214/AJR.14.12939

49. Giganti F, Coppola A, Ambrosi A, Ravelli S, Esposito A, Freschi M, et al. Apparent diffusion coefficient in the evaluation of side-specific extracapsular extension in prostate cancer: development and external validation of a nomogram of clinical use. Urol Oncol Semin Orig Investig 2016; 34: 291. e9-17. doi: 10.1016/j.urolonc.2016.02.015

50. Barrett T, Priest AN, Lawrence EM, Goldman DA, Warren AY, Gnanapragasam $\mathrm{VJ}$, et al. Ratio of tumor to normal prostate tissue apparent diffusion coefficient as a method for quantifying DWI of the prostate. AJR Am J Roentgenol 2015; 205: W585-93. doi: 10.2214/AJR.15.14338

51. Rosenkrantz AB, Neil J, Kong X, Melamed J, Babb JS, Taneja SS, et al. Prostate cancer: comparison of 3D T2-weighted with conventional 2D T2-weighted imaging for image quality and tumor detection. AJR Am J Roentgenol 2010; 194: 446-52. doi: 10.2214/AJR.09.3217

52. Itatani R, Namimoto T, Takaoka H, Katahira K, Morishita S, Kitani K, et al. Extracapsular extension of prostate cancer: diagnostic value of combined multiparametric magnetic resonance imaging and isovoxel 3-dimensional T2-weighted imaging at 1.5 T. J Comput Assist Tomogr 2015; 39: 37-43. doi: 10.1097/RCT.0000000000000172

53. Liberatore $M$, Delongchamps NB, Eiss $D$, Beuvon $F$, Zerbib $M$, Flam $T$, et al. Endorectal 3D T2-weighted $1 \mathrm{~mm}$-slice thickness MRI for prostate cancer staging at 1.5Tesla: should we reconsider the indirects signs of extracapsular extension according to the D'Amico tumor risk criteria? Eur J Radiol 2011; 81: e591-7. doi: 10.1016/j.ejrad.2011.06.056

54. Jäderling $F$, Nyberg $T$, Öberg $M$, Carlsson $S$, Skorpil $M$, Blomqvist $L$. Accuracy in local staging of prostate cancer by adding a three-dimensional T2-weighted sequence with radial reconstructions in magnetic resonance imaging. Acta Radiol Open 2018; 7: 205846011875460. doi $10.1177 / 2058460118754607$ 
55. Peng $Y$, Schmid-Tannwald C, Wang S, Antic T, Jiang $Y$, Eggener $S$, et al. Seminal vesicle invasion in prostate cancer: evaluation by using multiparametric endorectal MR imaging. Radiology 2013; 267: 797-806. doi: 10.1148/radiol.13121319

56. Chan KK, Choi D, Byung KP, Ghee YK, Hyo KL. Diffusion-weighted MR imaging for the evaluation of seminal vesicle invasion in prostate cancer: initial results. J Magn Reson Imaging 2008; 28: 963-9. doi: 10.1002/jmri.21531

57. Potter SR, Epstein JI, Partin AW. Seminal vesicle invasion by prostate cancer: prognostic significance and therapeutic implications. Rev Urol 2000; 2: 1905. PMID: 16985773

58. Wang L, Hricak H, Kattan MW, Chen HN, Kuroiwa K, Eisenberg HF, et al. Prediction of seminal vesicle invasion in prostate cancer: incremental value of adding endorectal MR imaging to the Kattan nomogram. Radiology 2007; 242: 182-8. doi: 10.1148/radiol.2421051254

59. Grivas N, Hinnen K, de Jong J, Heemsbergen W, Moonen L, Witteveen T, et al. Seminal vesicle invasion on multi-parametric magnetic resonance imaging: correlation with histopathology. Eur J Radio/ 2018; 98: 107-12. doi: 10.1016/j.ejrad.2017.11.013

60. Roethke M, Kaufmann S, Kniess M, Ketelsen D, Claussen CD, Schlemmer HP et al. Seminal vesicle invasion: accuracy and analysis of infiltration pattern with high-spatial resolution T2-weighted sequences on endorectal magnetic resonance imaging. Urol Int 2014; 92: 294-9. doi: 10.1159/000353968

61. Ohori M, Scardino PT, Lapin SL, Seale-Hawkins C, Link J, Wheeler TM. The mechanisms and prognostic significance of seminal vesicle involvement by prostate cancer. Am J Surg Pathol 1993; 17: 1252-61. doi: 10.1097/00000478-199312000-00006

62. Jung DC, Lee HJ, Kim SH, Choe GY, Lee SE. Preoperative MR imaging in the evaluation of seminal vesicle invasion in prostate cancer: pattern analysis of seminal vesicle lesions. J Magn Reson Imaging 2008; 28: 144-50. doi: 10.1002/jmri.21422

63. Soylu FN, Peng Y, Jiang Y, Wang S, Schmid-Tannwald C, Sethi I, et al. Seminal vesicle invasion in prostate cancer: evaluation by using multiparametric endorectal MR imaging. Radiology 2013; 267: 797-806. doi: 10.1148/ radiol.13121319

64. Gofrit ON, Zorn KC, Taxy JB, Zagaja GP, Steinberg GD, Shalhav AL. The dimensions and symmetry of the seminal vesicles. J Robot Surg 2009; 3: 29-33. doi: 10.1007/s11701-009-0134-x

65. Saliken JC, Gray RR, Donnelly BJ, Owen R, White L, Ali-Ridha N, et al. Extraprostatic biopsy improves the staging of localized prostate cancer. Can Assoc Radiol J 2000; 51: 114-20. PMID: 10786920

66. Barrett T, Tanner J, Gill AB, Slough RA, Wason J, Gallagher FA. The longitudinal effect of ejaculation on seminal vesicle fluid volume and whole-prostate ADC as measured on prostate MRI. Eur Radiol 2017; 27: 5236-43. doi: $10.1007 /$ s00330-017-4905-x

67. Medved M, Sammet S, Yousuf A, Oto A. MR Imaging of the prostate and adjacent anatomic structures before, during, and after ejaculation: qualitative and quantitative evaluation. Radiology 2014; 271: 452-60. doi: 10.1148/ radiol.14131374

68. Shin T, Kaji Y, Shukuya T, Nozaki M, Soh S, Okada H. Significant changes of T2 value in the peripheral zone and seminal vesicles after ejaculation. Eur Radiol 2018; 28: 1009-15. doi: 10.1007/s00330-017-5077-4

69. Kabakus IM, Borofsky S, Mertan FV, Greer M, Daar D, Wood BJ, et al. Does abstinence from ejaculation before prostate MRI improve evaluation of the seminal vesicles? AJR Am J Roentgenol 2016; 207: 1205-9. doi: 10.2214/ AJR.16.16278

70. McMahon CJ, Rofsky NM, Pedrosa I. Lymphatic metastases from pelvic tumors: anatomic classification, characterization, and staging. Radiology 2010; 254: 31-46. doi: 10.1148/radiol.2541090361

71. Joniau S, Van den Bergh L, Lerut E, Deroose CM, Haustermans K, Oyen R, et al. Mapping of pelvic lymph node metastases in prostate cancer. Eur Urol 2013; 63: 450-8. doi: 10.1016/j.eururo.2012.06.057

72. Briganti A, Suardi N, Capogrosso P, Passoni N, Freschi M, Di Trapani E, et al. Lymphatic spread of nodal metastases in high-risk prostate cancer: the ascending pathway from the pelvis to the retroperitoneum. Prostate 2012; 72: 186-92. doi: 10.1002/pros.21420

73. Barentsz JO, Severens JL, Hoogeveen YL, Hövels AM, Adang EM, Jager GJ, et al. The diagnostic accuracy of CT and MRI in the staging of pelvic lymph nodes in patients with prostate cancer: a meta-analysis. Clin Radiol 2008; 63: 387-95. doi: 10.1016/j.crad.2007.05.022
74. Vag T, Heck MM, Beer AJ, Souvatzoglou M, Weirich G, Holzapfel K, et al. Preoperative lymph node staging in patients with primary prostate cancer: comparison and correlation of quantitative imaging parameters in diffusion-weighted imaging and 11C-choline PET/CT. Eur Radiol 2014; 24: 1821-6. doi: 10.1007/s00330-014-3240-8

75. Eiber M, Beer AJ, Holzapfel K, Tauber R, Ganter C, Weirich G, et al Preliminary results for characterization of pelvic lymph nodes in patients with prostate cancer by diffusion-weighted MR-imaging. Invest Radiol 2010; 45: 15-23. doi: 10.1097/RLI.0b013e3181bbdc2f

76. Beer AJ, Eiber M, Souvatzoglou M, Holzapfel K, Ganter C, Weirich G, et al. Restricted water diffusibility as measured by diffusion-weighted MR imaging and choline uptake in 11C-choline PET/CT are correlated in pelvic lymph nodes in patients with prostate cancer. Mol Imaging Biol 2011; 13: 352-61. doi: 10.1007/s11307-010-0337-6

77. Vallini V, Ortori S, Boraschi P, Manassero F, Gabelloni M, Faggioni L, et al. Staging of pelvic lymph nodes in patients with prostate cancer: usefulness of multiple b value SE-EPI diffusion-weighted imaging on a 3.0 T MR system. Eur J Radiol Open 2016; 3: 16-21. doi: 10.1016/j.ejro.2015.11.004

78. Roy C, Bierry G, Matau A, Bazille G, Pasquali R. Value of diffusion-weighted imaging to detect small malignant pelvic lymph nodes at $3 \mathrm{~T}$. Eur Radiol 2010; 20: 1803-11. doi: 10.1007/s00330-010-1736-4

79. Caglic I, Barrett T. Diffusion-weighted imaging (DWI) in lymph node staging for prostate cancer. Transl Androl Urol 2018; 7: 814-23. doi: 10.21037/ tau.2018.08.04

80. Abdel Razek AAK, Soliman NY, Elkhamary S, Alsharaway MK, Tawfik A. Role of diffusion-weighted MR imaging in cervical lymphadenopathy. Eur Radiol 2006; 16: 1468-77. doi: 10.1007/s00330-005-0133-x

81. Muenzel D, Duetsch S, Fauser C, Slotta-Huspenina J, Gaa J, Rummeny EJ, et al. Diffusion-weighted magnetic resonance imaging in cervical lymphadenopathy: report of three cases of patients with bartonella henselae infection mimicking malignant disease. Acta Radiol 2009; 50: 914-6. doi: 10.1080/02841850903061445

82. Kwee TC, Takahara T, Luijten PR, Nievelstein RAJ. ADC measurements of lymph nodes: inter- and intra-observer reproducibility study and an overview of the literature. Eur J Radiol 2010; 75: 215-20. doi: 10.1016/j. ejrad.2009.03.026

83. Braithwaite AC, Dale BM, Boll DT, Merkle EM. Short- and midterm reproducibility of apparent diffusion coefficient measurements at 3.0-T diffusion-weighted imaging of the abdomen. Radiology 2009; 250: 459-65. doi: $10.1148 /$ radiol.2502080849

84. Rosenkrantz AB, Oei M, Babb JS, Niver BE, Taouli B. Diffusion-weighted imaging of the abdomen at 3.0 Tesla: image quality and apparent diffusion coefficient reproducibility compared with 1.5 Tesla. J Magn Reson Imaging 2011; 33: 128-35. doi: 10.1002/jmri.22395

85. Sadinski M, Medved M, Karademir I, Wang S, Peng Y, Jiang Y, et al. Shortterm reproducibility of apparent diffusion coefficient estimated from diffusion-weighted MRI of the prostate. Abdom Imaging 2015; 40: 2523-8. doi: 10.1007/s00261-015-0396-x

86. von Below C, Daouacher G, Wassberg C, Grzegorek R, Gestblom C, Sörensen J, et al. Validation of $3 \mathrm{~T} \mathrm{MRI} \mathrm{including} \mathrm{diffusion-weighted} \mathrm{imaging}$ for nodal staging of newly diagnosed intermediate- and high-risk prostate cancer. Clin Radiol 2016; 71: 328-34. doi: 10.1016/j.crad.2015.12.001

87. Sushentsev N, Martin H, Rimmer Y, Barrett T. Added value of diffusionweighted MRI for nodal radiotherapy planning in pelvic malignancies. Clin Transl Oncol 2019. [Epub ahead of print]. doi.org/10.1007/s12094-01902068-0

88. Zacharias C, Kunder C, Giesel F, Daniel B, Hatami N, Harrison C, et al. Gallium 68 PSMA-11 PET/MR imaging in patients with intermediate- or high-risk prostate cancer. Radiology 2018; 288: 495-505. doi: org/10.1148/ radiol.2018172232

89. Freitag MT, Radtke JP, Hadaschik BA, Kopp-Schneider A, Eder M, Kopka K, et al. Comparison of hybrid 68Ga-PSMA PET/MRI and 68Ga-PSMA PET/CT in the evaluation of lymph node and bone metastases of prostate cancer. Eur J Nucl Med Mol Imaging 2016; 43: 70-83. doi: 10.1007/s00259-015-3206-3

90. Baltzer P, Kenner L, Hartenbach M, Mitterhauser M, Goldner GM, Grahovac $M$, et al. PSMA Ligand PET/MRI for primary prostate cancer: staging performance and clinical impact. Clin Cancer Res 2018; 24: 6300-7. doi: 10.1158/1078-0432.ccr-18-0768 
91. Harisinghani MG, Barentsz J, Hahn PF, Deserno WM, Tabatabaei S, van de $\mathrm{Kaa} \mathrm{CH}$, et al. Noninvasive detection of clinically occult lymph-node metastases in prostate cancer. N Eng/ J Med 2003; 348: 2491-9. doi: 10.1056/ NEJMoa022749

92. Birkhäuser FD, Studer UE, Froehlich JM, Triantafyllou M, Bains L, Petralia $\mathrm{G}$, et al. Combined ultrasmall superparamagnetic particles of iron oxideenhanced and diffusion-weighted magnetic resonance imaging facilitates detection of metastases in normal-sized pelvic lymph nodes of patients with bladder and prostate cancer. Eur Urol 2013; 64: 953-60. doi: 10.1016/j. eururo.2013.07.032

93. Thoeny HC, Triantafyllou M, Birkhaeuser FD, Froehlich JM, Tshering DW, Binser $\mathrm{T}$, et al. Combined ultrasmall superparamagnetic particles of iron oxide-enhanced and diffusion-weighted magnetic resonance imaging reliably detect pelvic lymph node metastases in normal-sized nodes of bladder and prostate cancer patients. Eur Urol 2009; 55: 761-9. doi: 10.1016/j. eururo.2008.12.034

94. Fortuin AS, Brüggemann R, van der Linden J, Panfilov I, Israël B, Scheenen TWJ, et al. Ultra-small superparamagnetic iron oxides for metastatic lymph node detection: back on the block. Wiley Interdiscip Rev Nanomedicine Nanobiotechnology 2018; 10: e1471. doi: 10.1002/wnan.1471

95. National Institute for Health and Care Excellence. Prostate cancer: diagnosis and management, clinical guideline [CG175], 2014. [cited 2019 March 28]. Available at: http://www.nice.org.uk/guidance/cg175/chapter/1-recommendations

96. Shen G, Deng H, Hu S, Jia Z. Comparison of choline-PET/CT, MRI, SPECT, and bone scintigraphy in the diagnosis of bone metastases in patients with prostate cancer: a meta-analysis. Skeletal Radiol 2014; 43: 1503-13. doi: 10.1007/s00256-014-1903-9

97. Padhani AR, Koh D-M, Collins DJ. Whole-body diffusion-weighted MR imaging in cancer: current status and research directions. Radiology 2011; 261: 700-18. doi: 10.1148/radiol.11110474

98. Padhani AR, Lecouvet FE, Tunariu N, Koh D-M, De Keyzer F, Collins DJ, et al. METastasis Reporting and Data System for Prostate Cancer: practical guidelines for acquisition, interpretation, and reporting of whole-body magnetic resonance imaging-based evaluations of multiorgan involvement in advanced prostate cancer. Eur Urol 2017; 71: 81-92. doi: 10.1016/j. eururo.2016.05.033

99. Lecouvet FE, El Mouedden J, Collette L, Coche E, Danse E, Jamar F, et al. Can whole-body magnetic resonance imaging with diffusion-weighted imaging replace Tc $99 \mathrm{~m}$ bone scanning and computed tomography for single-step detection of metastases in patients with high-risk prostate cancer? Eur Urol 2012; 62: 68-75. doi: 10.1016/j.eururo.2012.02.020

100. Pasoglou V, Michoux N, Peeters F, Larbi A, Tombal B, Selleslagh T, et al. Whole-body 3D T1-weighted MR imaging in patients with prostate cancer: feasibility and evaluation in screening for metastatic disease. Radiology 2015; 275: 155-66. doi: 10.1148/radiol.14141242

101. Larbi A, Pasoglou V, Triqueneaux P, Cyteval C, Tombal B, Omoumi P, et al. Whole-body MRI to assess bone involvement in prostate cancer and multiple myeloma: comparison of the diagnostic accuracies of the T1, short tau inversion recovery (STIR), and high b-values diffusion-weighted imaging (DWI) sequences. Eur Radiol 2018. [Epub ahead of print]. doi: 10.1007/ s00330-018-5796-1 